\title{
La torre del Rey de Oropesa Un modelo de fortificación renacentista
}

\author{
Santiago Lillo Giner ${ }^{a}$, Pablo Rodríguez-Navarro ${ }^{\text {b }}$ \\ Instituto de Restauración del Patrimonio, Universitat Politècnica de València, Valencia, Spain \\ asanlilgi@ega.upv.es, rodriguez@upv.es
}

\begin{abstract}
The tower named "del Rey" is one of the most clear examples of the fortification called "de transición" in the area of the eastern spanish coast that was, as in the present case, through the addition of new technological resources, a model of the adaptation of the old medieval defensive types to a new conception of the military architecture that appeared as a result of the development of the artillery.

Built by Joan de Cervelló in 1534 in Punta de les Lances of Oropesa (Castellón), an especially vulnerable emplacement to the attacks of the barbary pirates of the time, it owes its origin to a tower built in 1413th year from that It takes its rectangular shape and that it be attached, forming a soffit on the lower floors on which it supports the new stonework. Starting from its historical, geometric and constructive study arises as the main objective of this communication the generation of necessary metadata and three-dimensional models for the interpretation and effective enhancement of the tower. To do so, we propose the use of the most advanced technical resources in the area of graphical representation that will allow us to get three-dimensional photo-realistic models, incorporating both data of the existing building as those relating to their type, existing elements, etc. The obtained results intend to set, in connection with works belonging to other towers from the same historical and geographical scope, a database that can be used both as a reporting tool as a documentary source for future research.
\end{abstract}

Keywords: torre del Rey, Joan de Cervelló, fortification, torres vigía.

\section{Introducción}

La torre del Rey de Oropesa forma parte de la red defensiva de torres de vigilancia y defensa del litoral del Reino de Valencia construidas a partir del siglo XVI, integrada en el subsistema formado por cinco torres de los términos de Benicásim y Oropesa que protegía el acceso septentrional al triángulo económico y demográfico de Castellón, Onda y Villareal (Boira, 2007).

Se encuentra situada en el cabo de Oropesa, localidad perteneciente a la comarca de la Plana Alta de la provincia de Castellón, entre las torres vigía de la Sal de Cabanes al norte y la de la Corda en el mismo término de Oropesa, al sur.
Está considerada documentalmente como una torre de costa y destinada a la vigilancia y defensa del litoral, pero es tanta su capacidad que podría incluirse entre los llamados "pequeños castillos". Se trata de uno de los más singulares modelos en los que la estereotomía renacentista cobra su máxima dimensión. Por otra parte, su estructura abovedada, así como la incorporación de algunos elementos característicos de la arquitectura militar de la primera mitad del siglo XVI hacen que pueda considerarse como uno de los mejores ejemplos de la fortificación de transición de todo el litoral valenciano. En contraposición con otros modelos de la época, limitados a simples adaptaciones de 
tipologías canónicas, en la torre del Rey encontramos un compendio de las más modernas técnicas defensivas surgidas con el advenimiento de la artillería y que supusieron en su día un verdadero alarde de erudición tecnológica (Lillo, 2012). La presente investigación es un avance de los trabajos actualmente en fase de realización (ver nota al final).
La metodología de estudio se basa en la lectura constructiva del estado actual del edificio, sin que hayamos podido disponer de catas ni ensayos. Se ha elaborado una primera documentación gráfica que sirve de soporte a un análisis arquitectónico y de identificación de elementos y sistemas constructivos.

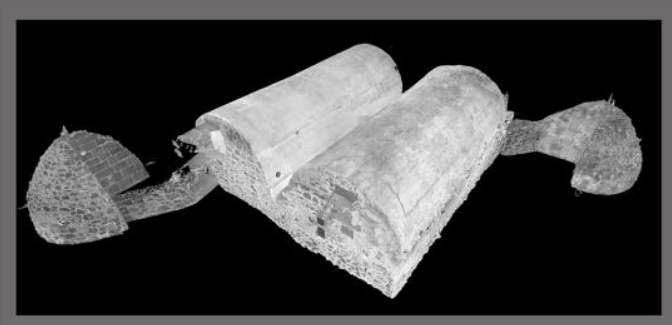

A

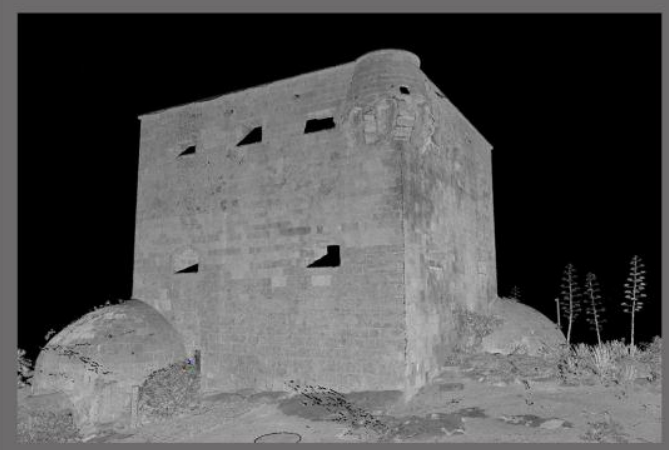

C

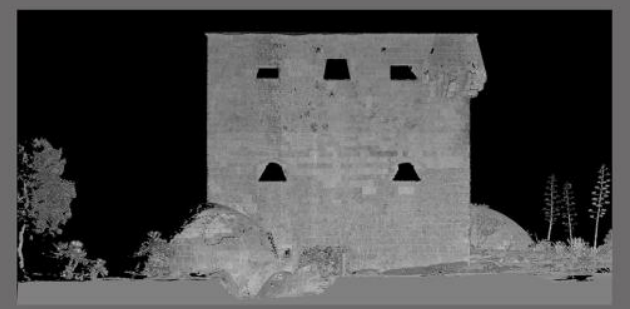

B

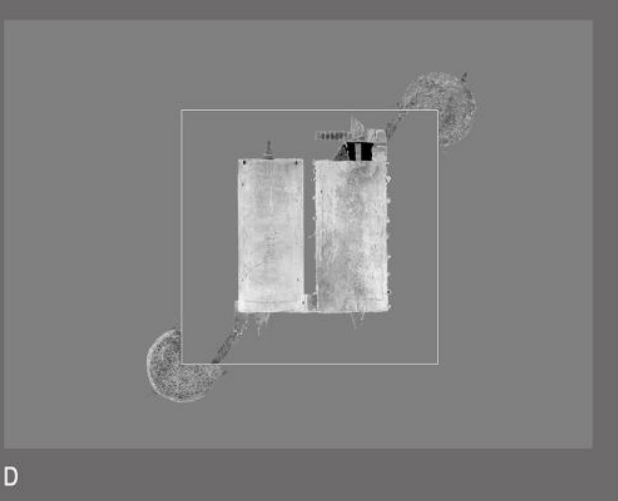

Fig. 1- Nubes de puntos obtenida mediante escáner láser 3D.a- Planta baja (interior). B-Alzado Noroeste. CPerspectiva. D- Planta baja. (Lillo-Verdiani)

Esta documentación se verá complementada con los trabajos que se están llevando a cabo en el marco del citado proyecto y que contemplan la obtención del levantamiento foto-realístico 3D (modelo virtual) a través de la obtención de nubes de puntos mediante escáner laser 3D y el procesado y compilación de los datos obtenidos en el trabajo de campo a través de software específico.

\section{Antecedentes}

Existen dos teorías contrapuestas acerca de la fecha de inicio de su construcción. Mientras que Sevillano Colom considera la torre como obra de 1413 (Sevillano, 1953), José María Doñate retrasa la fecha al siglo XVI (Doñate-Sebastiá, 1973). Por su parte Vicente Forcada concilia las dos teorías: la torre construida por Cervelló en 1534 habría sido edificada adosada a la anterior del siglo XV (Forcada-Martí, 1988).

Efectivamente, tras los ataques al vecino municipio de Torreblanca en 1397, la población de Oropesa solicitó a la corona la construcción de una torre vigía, petición que no pudo ser satisfecha hasta el año 1413 en que el Rey Fernando de Antequera autorizaba su ejecución. La obra propuesta no se limitaba únicamente a una torre para la vigilancia y defensa de la costa sino que venía complementada además con la construcción de una capilla o monasterio en la 
que pudiese permanecer una orden religiosa y una fortaleza cuyo recinto fuese capaz de proteger a la población en caso de ataque. Aunque en la actualidad sólo permanece en pie la primera, existen ciertos datos que nos permiten suponer que sí llegaron a edificarse, aunque con posterioridad, tanto el edificio religioso como el civil.

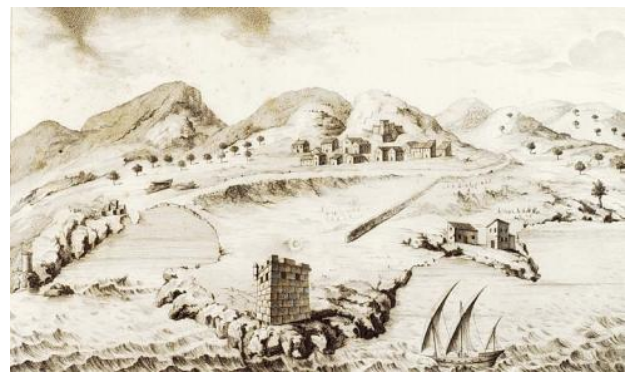

Fig. 2- Vista de Oropesa (T. L. Enguídanos, 1791)

En primer lugar, en las "Justas poéticas" del veedor de la Costa Catalá de Valeriola se hace mención de la presencia de una iglesia junto a la fortaleza: "...la torre de la Colomera y la torre del Barranch, paguiles en Oropesa, aon estiguí aquella nit y dormí en la fortalea es molt bona. Diumenge de matí, a 9, en haber pagat al alcait y els demés soldats, allí a una altra torre que está en altra montañeta, a un tir de escopeta del castell, y en la iglesia oy misa, que ya un altaret ab tres imagens de pedra marbre, de nostra Señora..." (ForcadaMartí, 1988).

En cuanto a la fortaleza, se han localizado ciertos vestigios de argamasa de mortero de cal en el entorno de la torre que parecen delatar la anterior presencia de un recinto rectangular en el ángulo suroeste y Norte de la torre. Estos restos podrían coincidir con el trazado que aparece en el grabado correspondiente al cabo de Oropesa (fig. 3) y que ilustra la narración de la toma de la fortaleza por parte de las tropas del Mariscal Suchet durante la guerra de la independencia de 1811, contenida en sus memorias (Suchet, 2008).

Finalmente, en el año 1534 Joan de Cervelló "retirado a Oropesa gastó veintiun mil ducados en labrar el fuerte que tenemos dicho (la torre del Rey)“ (Escolano, 1972), cantidad que Sebastián García reduce a quince mil al tiempo que destaca el hecho de que "raramente la iniciativa particular se ocupaba de edificar una fortaleza como hizo el Sr. de Oropesa D. Juan de Cervelló en 1534 con gasto de 15.000 ducados..." (García-Martínez, 1972).

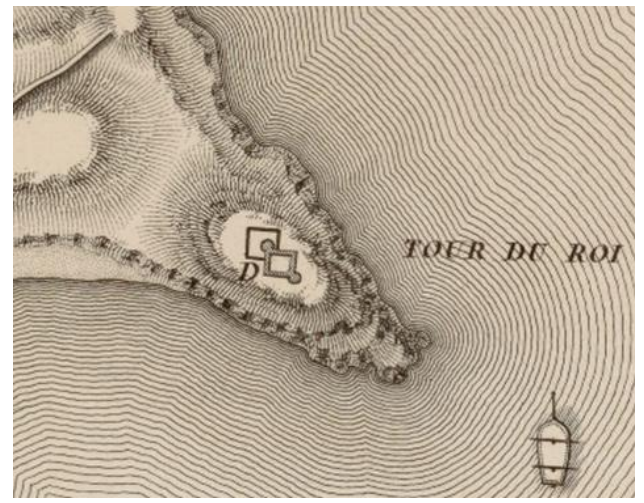

Fig. 3- Plan du fort d'Oropesa et de la Tour du Roi assiegés et pris en octobre 1811 (E. Collin, 1828)

En cualquier caso su autoría queda ratificada por los testimonios de dos de las personas que participaron en el pleito que tuvo Laura de Cervelló, nieta de Don Juan, con los acreedores de su padre. En primer lugar el de Jerónimo de Cabanilles en 1586: "La qual dita torre e fortalea axí mateix sap ell testimoni e té plena notícia e sabiduria que aquella fonch edificada $\mathrm{e}$ construida per lo dit quondam don Joan Servelló, pare del dit don Pedro Servelló, de sos propris diners, y per dita rahó diu ell dit testimoni tenir notícia que recaygué en bens y herència de aquells" (ARV, f. 40v) y dos años después el de Joan Martí cuando afirma de Joan de Cervelló que "aquell feu y edificà dita torre de Oropesa en dit capítol mencionada de fonament, que sols hi havia en dita torre una bodegueta, y axí aquell edifficà y feu dita fortalesa" (ARV, f. 286v).

A este proyecto, y probablemente gracias al prestigio que con él alcanzó como ingeniero en la corte real, se sucedieron varias intervenciones para actualizar las fortalezas de Alicante, Benidorm y Peñíscola. 


\section{Descripción general}

Como se ha dicho, la torre está constituida por la superposición de dos torres adosadas, de forma que la primera se establece como el intradós de la segunda, conformando un doble paramento hasta la cota de la planta primera.

Respecto a la torre de 1413 , se trata de una edificación de planta cuadrada y cuerpo prismático de $13,20 \mathrm{~m}$. de lado. Contaba probablemente con dos plantas principales y una auxiliar.

La planta baja queda dividida en dos espacios iguales por un muro intermedio. Los espacios resultantes se cubren por medio de bóvedas de cañón. De la planta principal queda tan sólo el posible vestigio de una almena entre los sillares de la nueva torre que hace suponer un remate almenado situado al nivel del primer piso de la torre actual.

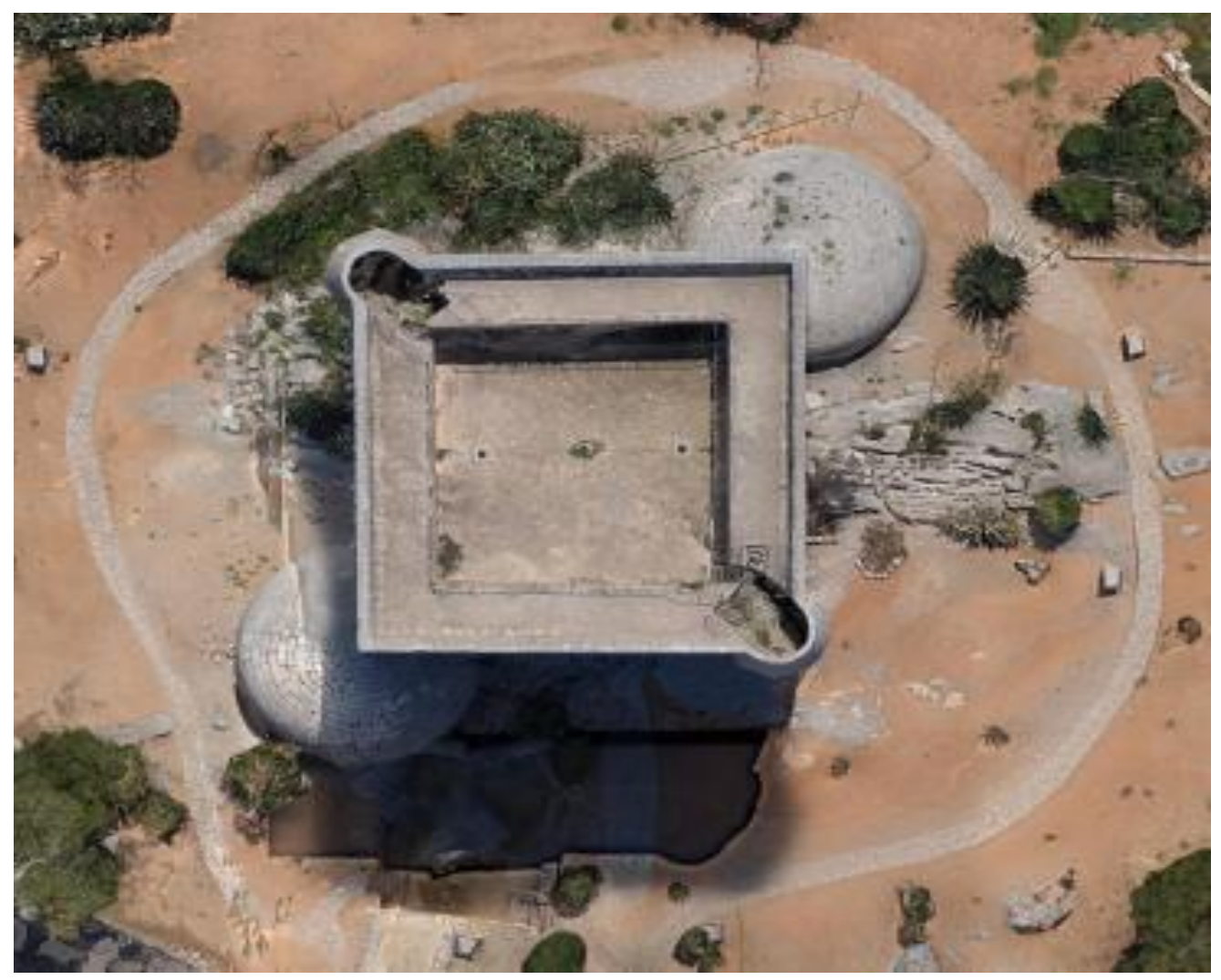

Fig. 4- Planta aérea obtenida mediante fotogrametría (Rodríguez-Navarro).

La torre del siglo XVI se desarrolla en tres alturas sin talud, de forma que la torre primitiva se encuentra circundada en planta baja y parte de la principal por los muros de la nueva, que alcanzan una altura total de $15^{\prime} 70 \mathrm{~m}$. La planta baja es de sección cuadrada de $17^{\prime} 40 \mathrm{~m}$. de lado.

En las esquinas opuestas de los ángulos noroeste y sureste se disponen dos casamatas de forma semiesférica para la defensa de la torre. En la planta principal el espacio interior se encuentra dividido en dos estancias cubiertas con bóveda de cañón apoyadas sobre los muros perimetrales y sobre el muro medianero central que se dispone perpendicularmente al de la planta baja.

El acceso desde la planta inferior se lleva a cabo a través de la escalera ubicada en el espacio 
comprendido entre los muros de ambas torres en el ángulo suroeste de la estancia situada a poniente.

La planta superior se encuentra totalmente descubierta y está destinada fundamentalmente a la defensa de la propia torre. Está rodeada de troneras con mirillas. En los ángulos noreste y suroeste, opuestos a las caponeras, se disponen dos garitones circulares sobre ménsulas gallonadas de piedra equipados también con troneras.

La planta de coronamiento está constituida por el camino de ronda y el pretil sin almenas. Se accede desde la planta alta a través de una pequeña escalera situada junto a uno de los garitones.

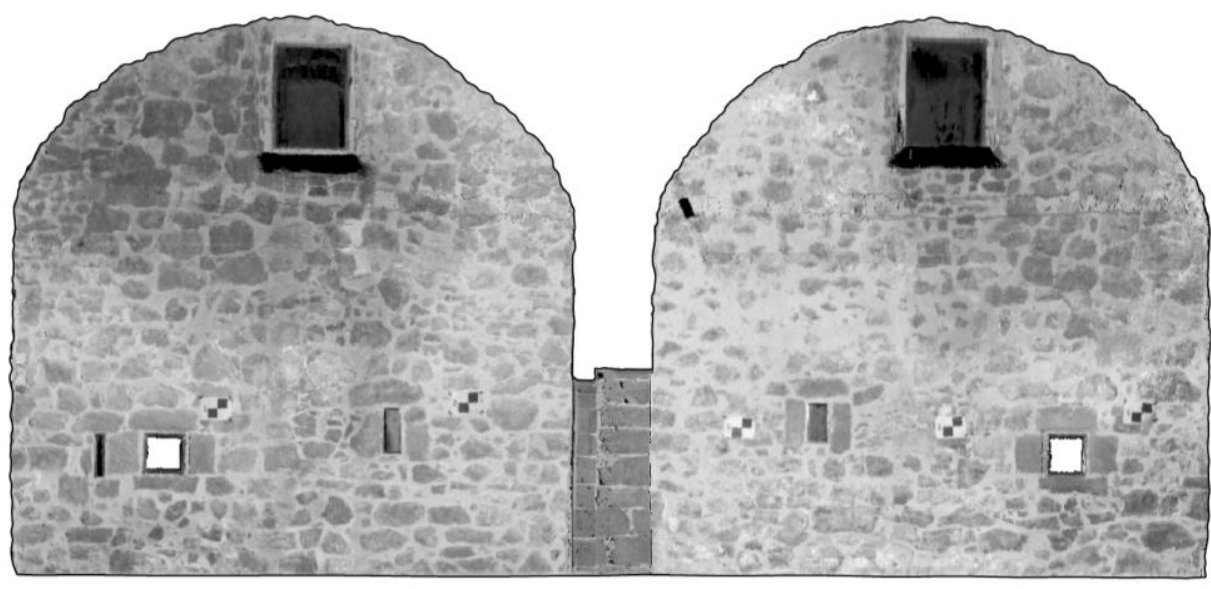

Fig. 5- Bóvedas de la planta principal. Sección transversal obtenida mediante nube de puntos (Elaboración gráfica: S. Lillo)

El conjunto se completa con una barbacana frente al portal entre el muro de poniente y la caponera noroeste y junto a ella un pequeño patio cerrado y el aljibe correspondiente a la primitiva torre.

\section{Elementos}

\subsection{Cimentación}

A pesar de que, como se ha indicado anteriormente, no se ha llevado a cabo la realización de catas, dadas las características del terreno de tipo rocoso cabe suponer, aunque no podemos determinar el nivel exacto de arranque, que el edificio asienta de forma directa sobre la roca, circunstancia que haría innecesaria la existencia de una base ataludada.

\subsection{Muros}

Los muros de la torre primitiva están construidos a base de mampostería de 1'50 $\mathrm{m}$. de espesor con posible refuerzo de sillería en esquinas y vanos. El muro de diafragma está realizado con el mismo material y cuenta con un espesor de 0'80 m. Los correspondientes a la torre nueva por su parte, que cuentan con un espesor total en planta baja de 3'60 m., son en su cara exterior de sillería de piedra caliza tanto en paramentos, caponeras y garitones superiores, mientras que en el interior se emplea la mampostería recibida con mortero de cal en paramentos, bóvedas, caponeras y escaleras y la piedra sillar para las troneras. En la planta principal el espesor de los muros se reduce hasta los 2'86 m.

\subsection{Bóvedas}

Como se ha dicho, tanto las estancias de la planta baja como las de la planta principal están cubiertas por sendas bóvedas de cañón de medio punto. Las correspondientes a la planta baja apoyan en los muros norte y sur y en el muro de diafragma central, mientras que las de la planta 
primera lo hacen en los muros este y oeste y en el muro central, lo que hace que queden contrapuestas. Todas están construidas a base de

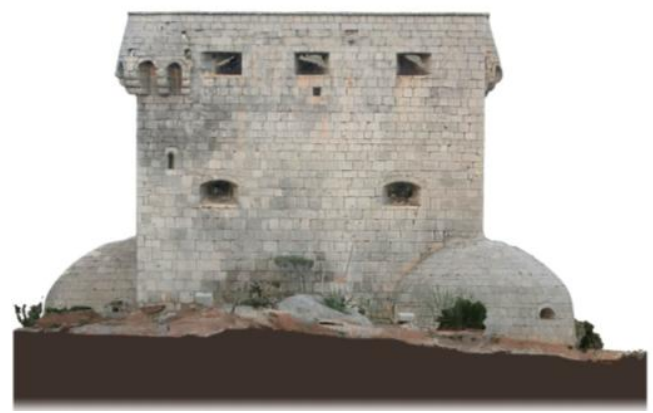

mampostería trabada mediante argamasa de mortero de cal. En el aljibe se utiliza el mismo tipo de cubierta.

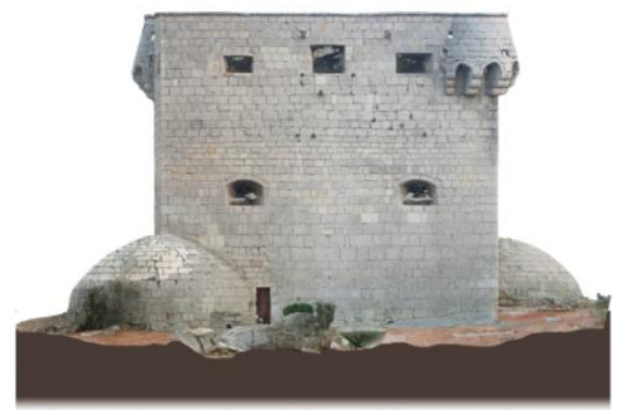

Fig. 6- Alzados Noreste y Noroeste obtenidos mediante fotogrametría (Rodríguez-Navarro).

\subsection{Vanos}

Constituyen uno de los elementos más característicos del edificio, puesto que configuran un complejo sistema de ejes y ángulos visuales trazados con el objetivo de barrer el perímetro completo de la torre y que parece haber sido fruto de un profundo estudio. La torre primitiva cuenta en su planta baja con dos vanos contrapuestos de mampostería y cantoneras de piedra sillar en cada una de las salas, de los cuales los orientados a oeste quedan cegados por los muros de la torre nueva.

En las esquinas de los ángulos noroeste y sureste se encuentran los accesos a las casamatas, ejecutados a base de sillería. En el vestíbulo generado entre los muros de las torres original y nueva se encuentran en el lado oeste el vano de acceso exterior, adintelado y ejecutado con sillares, al sur una abertura estrecha que discurre junto a la escalera y en el lado norte el acceso a una de las salas de la planta baja.

En la planta principal el acceso desde la escalera se lleva a cabo a través de un vano situado en el vértice suroeste del muro. Las dos salas de la planta quedan comunicadas por un portal de piedra sillar y arquería de medio punto de 1'50 m. de luz. Cada uno de los paramentos dispone de dos troneras con bocana abocinada equipadas con dos mirillas cada una que permiten ampliar el ángulo de tiro para una mejor defensa. Al exterior se abren con arco escarzano de sillería.
En la bóveda de la sala oeste se dispone una abertura rectangular que comunica la planta principal con la cubierta para el aprovisionamiento de la artillería. Por otra parte, la sala este queda comunicada con la escalera a través de un vano situado en el muro sur de la misma.

En la planta superior se disponen un total de doce troneras, tres en cada uno de los paramentos. Las situadas en los laterales tienen los ejes esviados. Todas ellas están equipadas, al igual que las de la planta inferior, con dos mirillas cada una, excepto las centrales de los muros este y oeste que se complementan con dos mirillas laterales suplementarias.

\subsection{Escaleras}

Se trata de una escalera de dos tramos en "L" que discurre, como se ha dicho, entre los muros de las torres vieja y nueva. La que da acceso a la planta principal se encuentra en el muro oeste, mientras que la que accede a la planta de cubiertas ocupa el lado sur. Está ejecutada a base de piedra sillar y cuenta con una anchura de 0'50 m.

\subsection{Elementos singulares, parapetos y remates superiores}

Las casamatas semiesféricas situadas en los ángulos opuestos noroeste y sureste de la planta baja constituyen sin lugar a dudas uno de los 
elementos más característicos e identificativos de la torre. Cubren un ángulo de $270^{\circ}$ y cuentan con radios de 5 y $2^{\prime} 70 \mathrm{~m}$. en la base. El espesor de 2'30 m. de sus muros, que disminuye en su perfil ascendente hasta los 0'50 m., hace que adquieran en el interior una forma ovoidal, mientras que en el exterior su forma es semiesférica, aunque no en toda su altura. Cada una de ellas dispone de tres troneras radiales con bocana abovedada.

En los ángulos opuestos a las casamatas se encuentran, a cota intermedia entre la planta superior y el paseo de ronda, dos garitones de sillería sobre ménsulas gallonadas del mismo material, a los que se accede desde éstas a través de pequeñas escaleras. Cada uno de ellos cuenta con tres troneras dotadas con mirillas.

El camino de ronda, al que se accede desde la planta exterior de cubiertas, constituye el último nivel transitable del edificio. Se encuentra delimitado por un pretil sin almenas interrumpido por la presencia de las garitas en las esquinas noreste y suroeste. El acceso se lleva a cabo desde la planta alta mediante una pequeña escala descubierta con arranque desde el pasillo de los garitones.

\section{Conclusiones}

El edificio, que ha sido restaurado con acierto y se encuentra en excelente estado de conservación, es propiedad en la actualidad de la Diputación Provincial de Castellón, y alberga el Museo de la Torre del Rey. Se encuentra además bajo la protección de la Declaración genérica del Decreto de 22 de abril de 1949, y la Ley 16/1985 sobre el Patrimonio Histórico Español.

Sin embargo, es necesario señalar una circunstancia que afecta tanto a este edificio como a gran parte de nuestro patrimonio construido, especialmente a aquellos erigidos en las zonas próximas a la costa, como consecuencia del actual modelo de desarrollo turístico. Efectivamente, la torre del Rey se encuentra en la actualidad rodeada de edificios de apartamentos cuya altura en relación con ella conlleva la pérdida del carácter referencial y estratégico, de hito costero, que durante varios siglos ha supuesto una de las características principales de las torres de vigía y defensa del litoral. Se hace por tanto necesario una vez más advertir sobre las amenazas que ponen en riesgo no únicamente la idiosincrasia de nuestro patrimonio construido, sino también su propia existencia, incidiendo en la importancia de protegerlo y conservarlo a través de su estudio, su conocimiento y su puesta en valor.

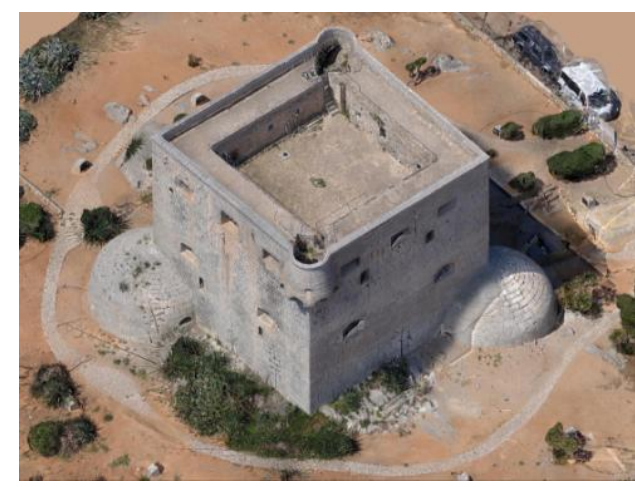

Fig. 7- Perspectiva isométrica obtenida mediante fotogrametría (Rodríguez-Navarro).

\section{Notas}

La presente aportación se ha realizado dentro del Proyecto I+D del Programa Estatal de Investigación Científica y Técnica de Excelencia, Subprograma Estatal de Generación de Conocimiento, financiado por el Ministerio de Economía y Competitividad, que lleva por título "Torres de vigía y defensa del litoral valenciano. Generación de metadatos y modelos 3D para su interpretación y efectiva puesta en valor", referencia HAR2013-41859-P.

\section{Referencias}

Arciniega, L. (1999). "Defensas a la antigua y a la moderna en el Reino de Valencia durante el siglo XVI" en Espacio, tiempo y forma. Serie VII. Historia del Arte. Valencia. pp. 61-94.

ARV. Real Audiencia. Procesos de Madrid, L.116, f. 40v, f. 286v.

Boira, J. V. (2007). Las torres del litoral valenciano. Generalitat Valenciana. Valencia. p. 78. 
Doñate-Sebastiá, J.M. (1973). "La torre del Rey" en Datos para la historia de Villarreal. Tomo III. Anubar. Vila-Real.

Escolano, G. (1972). Década primera de la historia de la insigne y coronada ciudad y Reino de Valencia : primera parte : [libros I y II]. Universitat de València. Valencia. p. 279.

Forcada-Martí, V. (1988). "La torre del Rey" en Boletín de la Sociedad Castellonense de Cultura. T. LXIV. Castellón. p. 371.

García-Martínez, S. (1972). "Bandolerismo, piratería y control de moriscos en Valencia durante el reinado de Felipe II" en Estudis: revista de historia moderna. n. 1. Universitat de València. Valencia. p. 101.

Lillo, S. (2012). La Ciudadela de Valencia. Origen, evolución y análisis gráfico. Tes-Doc. Universitat Politécnica de València. Valencia. p. 131.

Sevillano, F. (1953). Oropesa. Bosquejo histórico de Oropesa. Boletín de la Sociedad Castellonense de Cultura. Castellón.

Suchet, L. G. (2008). Memorias del mariscal Suchet, duque de La Albufera, sobre sus campañas en España, desde 1808 hasta 1814. Edición facsímil del original de 1828. IFC. Zaragoza. 